\title{
A SAR interferometric model for soil moisture
}

\author{
Francesco De Zan, Alessandro Parizzi, Pau Prats-Iraola and Paco López-Dekker
}

\begin{abstract}
There is a need for scattering models that link quantitatively SAR interferometric observables to soil moisture. In this work we propose a model based on plane waves and the Born approximation, deriving first the vertical complex wavenumbers in the soil as a function of geometrical and dielectric properties and successively the complex interferometric coherences. It is observed that soil moisture behaves on the phase in a similar way as tomography does, breaking the phase consistency in triplets of interferograms. The proposed model is validated with $L$-band airborne SAR data; preliminary inversion results based on interferogram triplets and coherence magnitudes are presented.
\end{abstract}

Index Terms-SAR Interferometry, soil moisture, coherence

\section{INTRODUCTION}

A relation between soil moisture and SAR interferometric phase has been recognized at least since 1989, when researchers working with SEASAT data at the Jet Propulsion Laboratory reported motions of a few centimeters that were linked to watering [1] and were clearly visible in one interferogram. This observation was made over agricultural fields in California and the proposed explanation was based on the effect of clay and salt expansion.

After SEASAT, interferometric features corresponding to moisture changes have been repeatedly observed in satellite SAR data (e.g. ERS, Envisat, ALOS, [2], [3], [4]) and in airborne and indoor experiments (e.g. [5], [6], [7]). Different explanations have been proposed, the most recurrent being the same provided originally in [1]: the vertical expansion of the fields, causing a differential effect in the radar line-of-sight.

This explanation is now under challenge, especially thanks to the works of Hensley and Morrison [8], [9]. Hensley has noted that the phase change is sometimes too large to be caused by a realistic deformation; Morrison has shown in an indoor experiment that the motion of the surface of the soil under study (sand) is much smaller than the motion of the corresponding C-band phase shift. The experimental observations require more satisfactory models.

In this paper we provide a new explanation of interferometric phases and coherences (Sect. II) and validate it with real Lband data over bare surfaces (Sect. III). We present first a very simple model; some obvious improvements and extensions are discussed in a dedicated section of the paper (Sect. IV). The main idea is that the differential propagation of the electromagnetic waves into the soil causes the interferometric effects, by affecting the vertical wavenumber in a thin layer close to the surface, in dependence on the moisture level.

So far the subject of soil moisture estimation with SAR has been addressed mainly with scatterometric/polarimetric

The authors are with DLR (German Aerospace Center), Oberpfaffenhofen, D-82230 Wessling, Germany. e-mail:francesco.dezan@dlr.de techniques, focusing on amplitude properties. For a review of the topic the reader is referred to [10]. Several relations have been developed to link the back-scattered amplitude to soil moisture levels. The main limitation for amplitude-based techniques was found to be surface roughness, which can influence backscatter independently of moisture. Differential methods can reduce the impact of roughness, for instance comparing the amplitude levels at two different frequencies, polarizations or incident angles.

Different from amplitudes, the full potential of interferometry for soil moisture retrieval is still largely unknown. It makes sense to investigate it, especially considering the typical precision of interferometric methods. Even if we were not interested in retrieving soil moisture levels with interferometric techniques, we could still be interested in predicting the effect on the interferograms and the coherence maps, at least to be able to compensate for it and isolate additional signals like change detection and deformation.

A link between interferometric phases and propagation in a wet soil was already mentioned in [1] but quickly discarded in favor of the mechanical expansion hypothesis. The authors of [11] showed -thanks to finite-difference time-domain modeling- that small scattering bodies in the soil or moisture gradients can produce phase and coherence variations. This is very similar to what we propose here, the difference is that we propose an analytical formulation. A simple analytical model has the advantage of helping the physical intuition even though it is usually less flexible compared to numerical simulation.

\section{PROPAGATION AND BACKSCATTER MODEL}

The model that we present consists of two distinct parts: the propagation of the incident wave in the soil and the backscatter. This artificial decoupling allows a simple analytical treatment and is justified by the assumption that the backscatter is generated only by small particles or small discontinuities in the soil. It is the very common first-order Born approximation (see, e.g. [12]): First, the incident field is computed as if the discontinuities were not present; second, the backscatter is estimated as a function of the computed incident field (it is actually a function of the total field, which is what makes the exact problem non-linear).

Concerning the propagation part, the soil is modeled as a uniform, lossy dielectric with a complex refraction index. The imaginary part of the refraction index is linked to the energy dissipation in the material (Ohmic losses). For the relation between the moisture parameters and the refraction index we refer to [13], which gives empirical curves for different soil types and frequencies (one example is reproduced in Fig. 2). The influence of the soil type is secondary, compared to moisture and frequency. 


\section{A. Oblique incidence on a lossy dielectric}

The geometry of the problem is 2-D, once the reference system has been properly aligned (Fig. 1 provides a sketch).

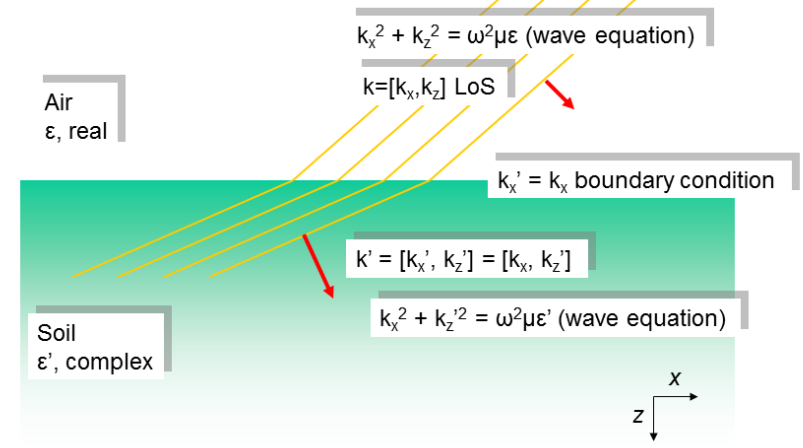

Fig. 1. The geometry of plane wave incidence and refraction on a flat lossy medium.

The $x z$ plane is the incidence plane (the azimuth direction $y$ is orthogonal to this plane) and the air-soil discontinuity is parallel to $x$ and is positioned at $z=0$. Note that the $z$-axis is directed downwards. The incident angle is $\theta_{\text {inc }}$.

The incident field is modeled as a plane wave, using the radar carrier frequency $c / \lambda$ as a reference. We follow the derivation in [14]. For the TE case (electric field orthogonal to the plane of incidence, $\mathrm{HH}$ polarization) the expressions for the incident and refracted (with primes) electric fields are:

$$
\begin{aligned}
E(\mathbf{r}) & =\hat{\mathbf{y}} E_{0} e^{-j k_{z} z} e^{-j k_{x} x} \\
E^{\prime}(\mathbf{r}) & =\hat{\mathbf{y}} \tau E_{0} e^{-j k_{z}^{\prime} z} e^{-j k_{x}^{\prime} x}
\end{aligned}
$$

The symbol $\hat{\mathbf{y}}$ indicates the unit vector in the $y$ direction. The complex amplitude of the incident field is $E_{0}$. The symbols $k_{x}$ and $k_{z}$ represent the complex wavenumbers in the two directions (with primes in the second medium). The Fresnel transmission coefficient for the TE case is

$$
\tau=2 k_{z} /\left(k_{z}+k_{z}^{\prime}\right) .
$$

The reflected field is not treated, because it does not contribute to the (monostatic) radar signal.

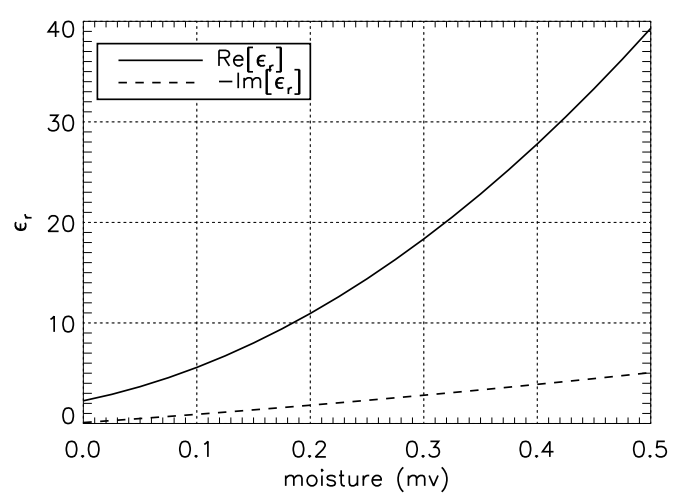

Fig. 2. The variation of the real and imaginary part of the relative dielectric constant according to [13]. The soil type is $51 \%$ sand and $13 \%$ clay and the frequency is $1.4 \mathrm{GHz}$.
Now the goal is to derive $k_{x}^{\prime}$ and $k_{z}^{\prime}$, i.e. the propagation in the second medium (the soil), for a given incidence geometry $\left(k_{x}=(2 \pi / \lambda) \sin \theta_{i n c}\right.$ and $\left.k_{z}=(2 \pi / \lambda) \cos \theta_{i n c}\right)$. The horizontal boundary conditions have to be satisfied:

$$
k_{x}^{\prime}=k_{x}
$$

To derive $k_{z}^{\prime}$ let us observe that the wave equations hold in the Fourier domain for both media: $k_{x}^{2}+k_{z}^{2}=\omega^{2} \epsilon \mu$ and $k_{x}^{\prime 2}+k_{z}^{\prime 2}=\omega^{2} \epsilon^{\prime} \mu$. The $\epsilon$ and $\epsilon^{\prime}$ are the two dielectric constants at the radar operating frequency: For the first medium (air) we can assume $\epsilon=\epsilon_{0}$. Finally, once $\theta_{i n c}$ and $k_{x}$ are set, $k_{z}^{\prime}$ is only a function of $\epsilon^{\prime}$ :

$$
k_{z}^{\prime}\left(\epsilon^{\prime}\right)=\sqrt{\omega^{2} \epsilon^{\prime} \mu-k_{x}^{2}} .
$$

Since the medium is lossy, $\epsilon^{\prime}$ and $k_{z}^{\prime}$ are complex numbers. The above equation has two solutions because of the ambiguity of the square root, and we chose the "physical" one, i.e. the one with a negative imaginary part. This corresponds to a wave that attenuates going downward, so that $\left|E^{\prime}(x, y, z)\right| \rightarrow 0$ when $z \rightarrow \infty$. The constant amplitude planes in the second medium are parallel to the interface, whereas the constant phase planes are not. The phase propagates in the direction of refraction, determined by $k_{x}$ and the real part of $k_{z}^{\prime}$. In practice the impedance contrast is so large that $k_{x}$ and the incident angle have almost no influence on $k_{z}^{\prime}\left(\epsilon^{\prime}\right)$.

This derivation has been conducted for the HH case. The VV case is much similar for what we are concerned. In particular, assuming isotropy of the medium, the propagation in the lossy medium $\left(k_{z}^{\prime}\right)$ is independent of the polarization.

The media are assumed to be isotropic and linear: these conditions are sufficient for reciprocity to hold [15], so that the propagation effects from the antenna to the scatterer and vice versa are identical. Because of this, the wavenumbers are simply doubled in the following to account for the two-way propagation.

\section{B. Interferometric model}

A focused pixel at range $r_{0}$ and azimuth $y_{0}$ can be modeled as a three-dimensional integral of elementary scatterer contributions from the soil, each with the proper propagation phase:

$$
\begin{array}{r}
p\left(r_{0}, y_{0}\right)=\int \xi^{\prime}(x, y, z) e^{-j 4 \pi \frac{r_{0}}{\lambda}-j 2 k_{x} x-j 2 k_{z}^{\prime} z} \\
\cdot h\left(r-r_{0}, y-y_{0}\right) d x d y d z
\end{array}
$$

The integral has support in the half-space with $z>0 ; h(r, y)$ is the end-to-end response of the SAR imaging system in the soil, and $\xi^{\prime}(x, y, z)$ is the three-dimensional scatterer distribution. The range $r$ in the soil can be approximated locally by

$$
r=r_{0}+x \sin \theta^{\prime}+z \cos \theta^{\prime},
$$

with $\theta^{\prime}$ being the refraction angle. Since the penetration is very limited, the variation of $\theta^{\prime}$ has almost no effect on the signal. Moreover, the ground-range and azimuth extensions of the resolution cell are constant at all depths. 
With the substitution

$$
\begin{gathered}
\xi(z)=\int \xi^{\prime}(x, y, z) e^{-j 4 \pi \frac{r_{0}}{\lambda}-j 2 k_{x} x} . \\
\cdot h\left(x \sin \theta^{\prime}+z \cos \theta^{\prime}, y-y_{0}\right) d x d y
\end{gathered}
$$

one can rewrite (6) to obtain the simple relation:

$$
p\left(r_{0}, y_{0}\right)=\int_{0}^{\infty} \xi(z) e^{-j 2 k_{z}^{\prime} z} d z
$$

This expression highlights that the only relevant direction for our case is $z$ (in particular the ground range $x$ is irrelevant because the propagation along the boundary will always be the same). A change in soil moisture will induce a change in $\epsilon^{\prime}$, thus in $k_{z}^{\prime}$, and consequently in the integral (9).

In the last expression $\xi(z)$ can be seen as a realization of a stochastic process, characterized by the property

$$
\mathbf{E}\left[\xi(z) \xi^{*}\left(z_{0}\right)\right]=f(z) \delta\left(z-z_{0}\right),
$$

where $f(z)$ is a vertical scattering density profile (a positive function).

The expected value of the interferogram between two images with different $k_{z}^{\prime}$ in the soil is computed as follows:

$$
\begin{aligned}
& I\left(\epsilon_{1}^{\prime}, \epsilon_{2}^{\prime}\right)=\mathbf{E}\left[p_{1} p_{2}^{*}\right] \\
& =\mathbf{E}\left[\iint \xi\left(z_{1}\right) e^{-j 2 k_{z 1}^{\prime} z_{1}} d z_{1}\left(\xi\left(z_{2}\right) e^{-j 2 k_{z 2}^{\prime} z_{2}} d z_{2}\right)^{*}\right] \\
& =\int_{0}^{\infty} f(z) e^{-j 2 k_{z 1}^{\prime} z}\left(e^{-j 2 k_{z 2}^{\prime} z}\right)^{*} d z
\end{aligned}
$$

making use of (9) and (10), which allows simplifying the double integral (the product of $p_{1}$ and $p_{2}$ ) to a single one. Each scatterer will interfere coherently only with itself, with the complex weighting given by the local phasor. The phase effect is thus a consequence of the differential propagation in the vertical direction.

In the particular case of a uniform vertical profile the integral (11) gives:

$$
I\left(\epsilon_{1}^{\prime}, \epsilon_{2}^{\prime}\right)=\frac{1}{2 j k_{z 1}^{\prime}-2 j k_{z 2}^{\prime}{ }^{*}}
$$

and the complex coherence results:

$$
\begin{aligned}
\gamma & =\frac{I\left(\epsilon_{1}^{\prime}, \epsilon_{2}^{\prime}\right)}{\sqrt{I\left(\epsilon_{1}^{\prime}, \epsilon_{1}^{\prime}\right) I\left(\epsilon_{2}^{\prime}, \epsilon_{2}^{\prime}\right)}} \\
& =\frac{2 j \sqrt{\operatorname{Im}\left(k_{z 2}^{\prime}\right) \operatorname{Im}\left(k_{z 1}^{\prime}\right)}}{k_{z 2}^{\prime}{ }^{*}-k_{z 1}^{\prime}}
\end{aligned}
$$

This expression gives both interferometric phases and coherences.

Figure 3 reports an example of coherence magnitudes as a function of master and slave moisture levels. Generally speaking, the more the two moisture values differ, the smaller is the coherence.

Similarly, Fig. 4 reports the interferometric phases. There is a very high sensitivity to small moisture changes. For example, a moisture change from $20 \%$ to $21 \%$ corresponds to $10 \mathrm{deg}$ interferometric phase variation. For the phases we are ignoring an additional contribution due to the complex transmission coefficient $\tau$ at the boundary. The phase of the transmission

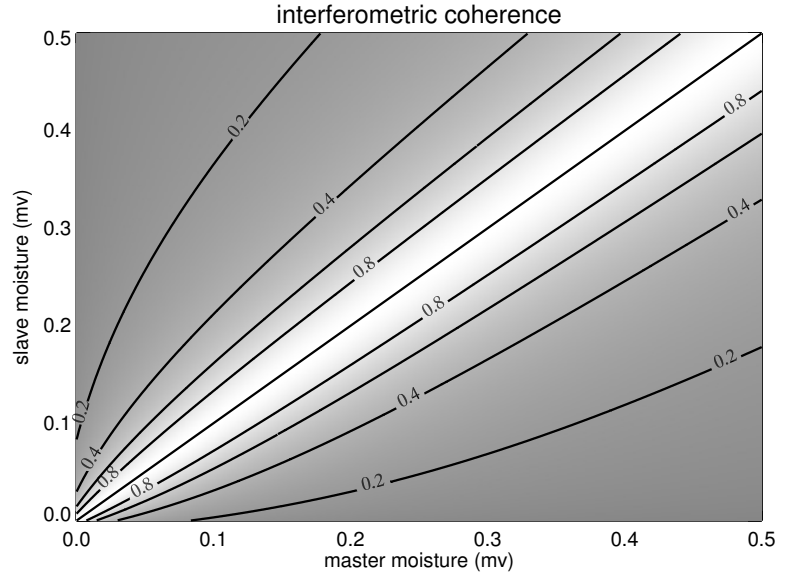

Fig. 3. The modulus of the coherence (14) as a function of soil moisture for a soil $51 \%$ sand and $13 \%$ clay acquired in L-band from and incidence of $45 \mathrm{deg}$ ( $\epsilon$ modeled following [13]).

coefficient does not affect the coherence moduli. Figure 5 reports the phase jump for the transmitted field at the interface for the TE un TM cases. As it was noted already in [1], the magnitude of this phase is very small compared to the variations observed in real data. It is also much smaller than the phases predicted by our model and it would be the only aspect to distinguish the $\mathrm{HH}$ and VV interferograms.

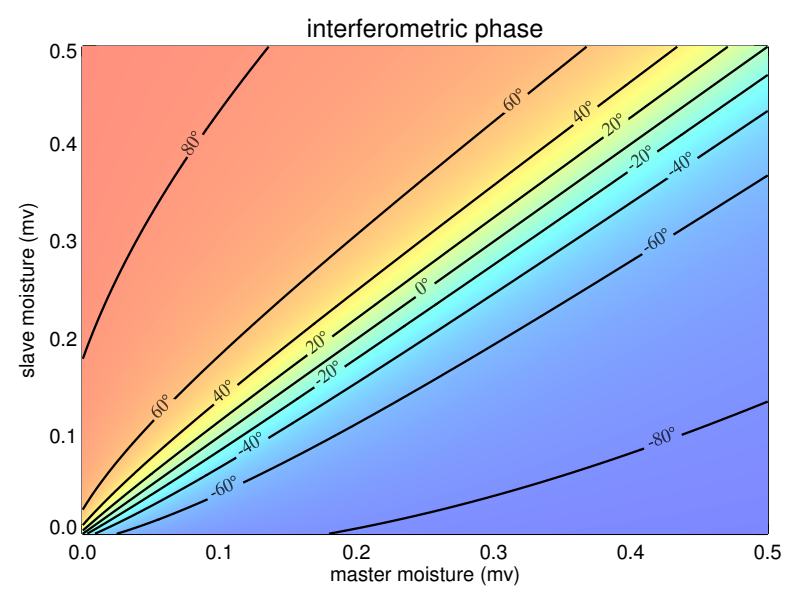

Fig. 4. The interferometric phases in degrees according to (14) as a function of soil moisture for a setting as in Fig. 3. The phase variation is non-linear.

According to the presented model, the interferometric phases have a non-linear behavior which can be understood examining Fig. 4: depending on the image taken as a reference, the total phase excursion looks larger or smaller. This is an indication that we are not seeing a single object, but more than one, similarly to what happens in tomography. Another way to see it, is to say that the covariance matrix of the acquisitions is not real, even after compensating for a free-space propagation term.

From the coherence expression (14) it follows that a mere change in the penetration depth does not change the interferometric phase. This is because a change in the penetration depth 


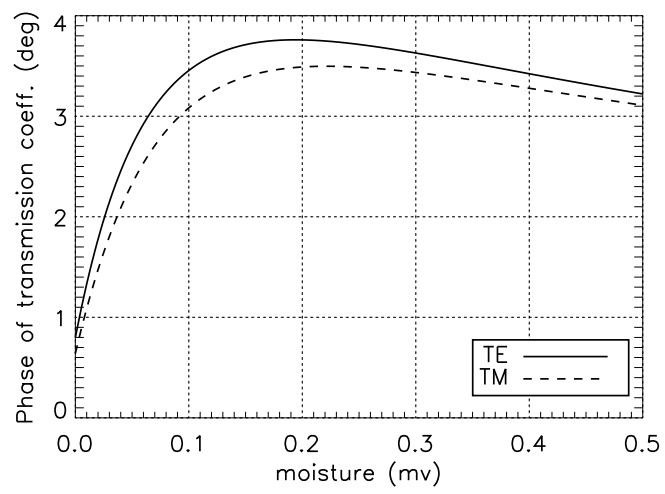

Fig. 5. The phase of the (one-way) transmission coefficient for different moisture levels and the parameters of Fig 3.

is linked to a change in the imaginary part of $k_{z}^{\prime}$ (remember the propagation is $\exp \left(-j k_{z}^{\prime} z\right)$ ), while a change in the vertical phase is linked to a change in the real part of $k_{z}^{\prime}$. If the real part of $k_{z}^{\prime}$ stays the same, so will the phase of the coherence, since the denominator in (14) becomes purely imaginary, like the numerator.

It is a common misconception that a pure change in the penetration depth can yield changes in the phase. One can think that the change in penetration depth between two acquisitions is equivalent to adding or subtracting scatterers. New deeper scatterers in the image with drier conditions are not correlated with the ones visible in the image with wetter conditions, so that finally they do not contribute to the interferometric phase but only to the coherence loss. The phase effect, as explained by this model, is essentially due to the common scatterers which are taken with different phases due to propagation effects. Penetration plays a role only in weighting the scatterers (hence the differential propagation phases) at different depths.

\section{VALIDATION WITH REAL DATA}

In this section we report some results obtained with data from the ESA AGRISAR campaign of 2006 [16]. The dataset comprises, among others, 12 SAR images acquired by the E-SAR L-band system of DLR and ground measurements of moisture. The test site is near the village of Demmin in northern Germany. The acquisitions span three months and a half, between April, 19th and August, 2nd (see Tab. I). Figure 7 displays as an example the differential interferometric phase of a pair of images in the dataset. As it is often the case, phase discontinuities follow the boundaries between different agricultural fields. Since vegetation is not modeled, we picked a corn field (field number 222 in [16]) which is expected to have a delayed growth. In-situ measurements of soil moisture are available only for neighboring fields, but the values are similar for different fields (see Fig. 12).

\section{A. Forward model}

We predict complex coherences using moisture values from one of the available fields, namely field number 101, using (5) and (14). Fig. 6 allows comparing predicted and

\begin{tabular}{|c|c|c|c|}
\hline Date & DoY(2006) & Date & $\operatorname{DoY}(2006)$ \\
\hline April, 19th & 109 & June, $13^{\text {th }}$ & 164 \\
\hline May, $3^{\text {rd }}$ & 123 & June, $21^{\text {st }}$ & 172 \\
\hline May, $11^{\text {th }}$ & 131 & July, $5^{\text {th }}$ & 186 \\
\hline May, $16^{\text {th }}$ & 136 & July, $12^{\text {th }}$ & 193 \\
\hline May, $24^{\text {th }}$ & 144 & July, $26^{\text {th }}$ & 207 \\
\hline June, $7^{\text {th }}$ & 158 & August, $2^{\text {nd }}$ & 214 \\
\hline
\end{tabular}

TABLE I

ACQUISITION DATES OF THE E-SAR IMAGES DURING THE AGRISAR CAMPAIGN OF 2006. THE SECOND COLUMN REPORTS THE CORRESPONDING DAY OF THE YEAR (DOY).

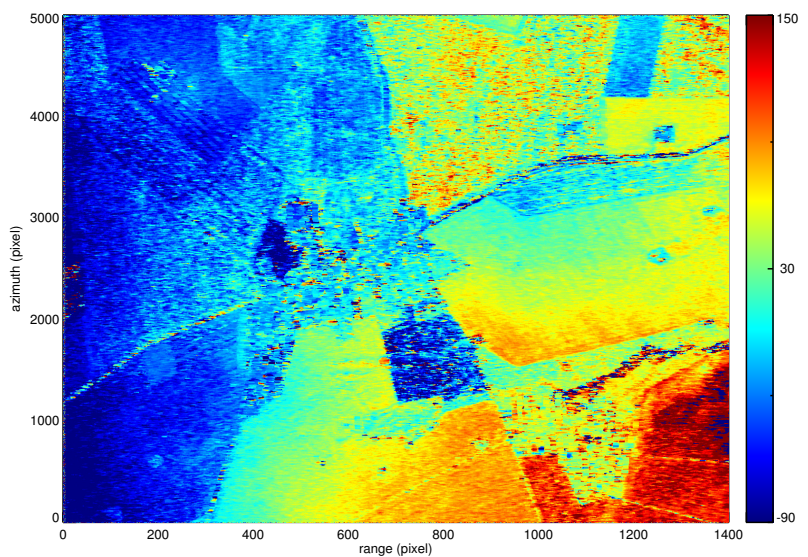

Fig. 7. The interferometric phase between May, 24th and June, 6th. Since the phase has not been calibrated, only relative effects are meaningful. Phases are expressed in degrees.

observed coherence magnitudes, using 8 images acquired between May, 11th and July, 12th. Each subfigure collects the coherences for a different master. The polarization channel is $\mathrm{HH}+\mathrm{VV}$; similar results are also obtained for $\mathrm{HH}$ and VV. In this time span the agreement is generally good except for the last image (July, 12th) which seems to be uncorrelated possibly due to corn growth.

Before and after the selected time span, the model is not valid: the observed coherence is generally low. Before May, 11th it is probably due to plowing, since the two available images are totally uncorrelated with the rest. From July, 12th on $(2$ more images) it might be that the corn has grown too much, and the model is too simple. Another indication of the loss of validity of our model is the polarimetric phase between the $\mathrm{HH}$ and VV channels. The Fresnel coefficients give a very small contribution, and consequently the polarimetric phase is expected to be very small. However this is observed only from May, 11th to June, 13th according to Fig. 9.

In order to compare the interferometric phases directly, one should start with a good calibrated phase and have no motion in the area of interest. In order to circumvent this problem, we introduce the concept of phase triplets which are immune to phase calibration and also provide an insight into 

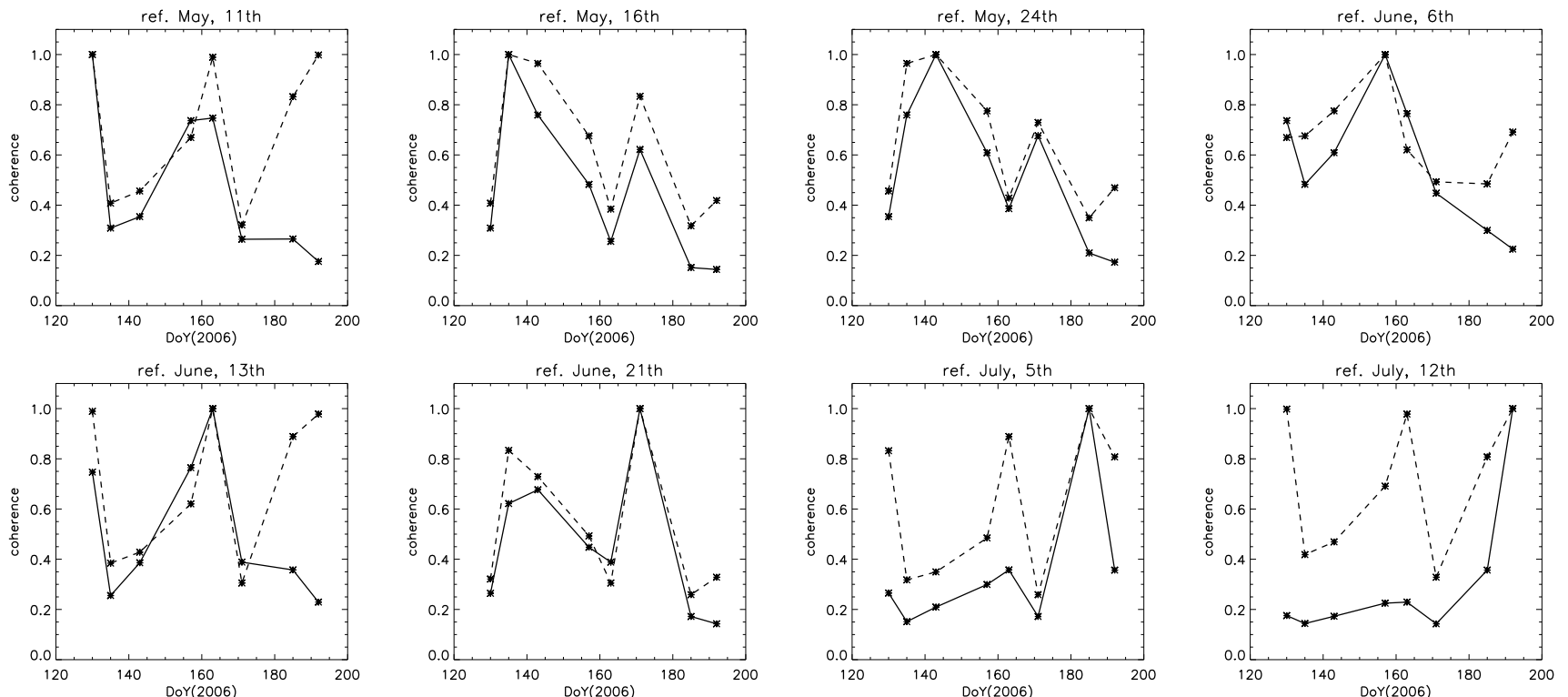

Fig. 6. Comparison between modeled (dashed lines) and observed (solid lines) coherences on field 222 of the AGRISAR 2006 dataset for the HH+VV polarization. The model seems to lose validity during July, possibly because of crop growth (corn).

the consequences of our model.

Phase triplets are phases of the three possible interferograms that can be made with three images. In the easiest case, one would expect that these phases match in such a way that having two of them allows to systematically predict the third, apart from decorrelation effects. For example, if $\varphi_{m, s}$ is the multilooked phase between master $m$ and slave $s$, with three images one would expect the triple difference

$$
\varphi_{1,2,3}=\varphi_{1,2}+\varphi_{2,3}-\varphi_{1,3}
$$

to be small (modulo $2 \pi$ ). Still, the model predicts systematic mismatches of tens of degrees. These are confirmed by observations in different polarimetric channels, as reported in Fig. 8.

It is worth noting that ignoring the small phase jump at the interface, i.e. the phase of the transmission coefficient, has no effect on the quantity $\varphi_{1,2,3}$, which remains unchanged when one image is multiplied by an arbitrary constant phase term.

\section{B. Moisture inversion experiment}

In this section we report about the inversion of moisture values from L-band SAR data. The inverse problem is always more delicate compared to the forward model, and we do not intend to validate an inversion algorithm, considering the limited data available.

There are several possibilities to invert the problem. Inversions based only on coherence magnitudes are prone to an ambiguity problem, since both increasing and decreasing soil moisture can produce the same coherence loss. The example we present is thus an inversion conducted using coherence magnitudes and phase triplets. Looking at Fig. 6 it seems that the model is valid until June, 21st: after that date an additional decorrelation term should be added, associated with crop growth. Because of this, the inversion results are based on six images between May, 11th and June, 21st.

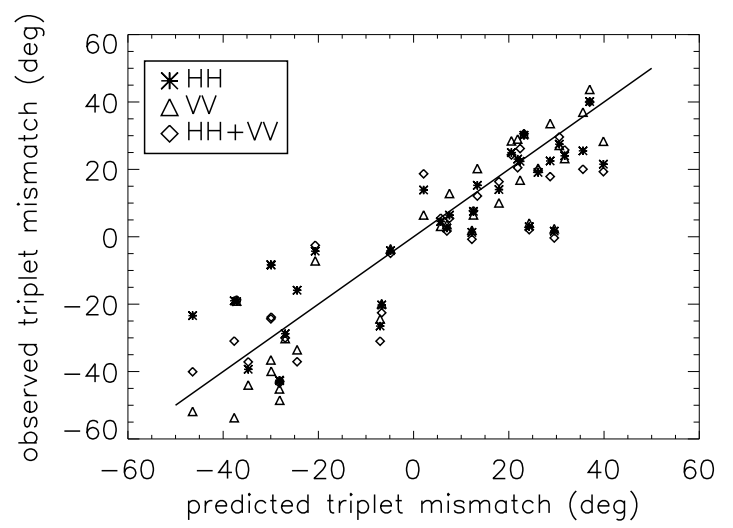

Fig. 8. Predicted and observed phase mismatches in all possible triplets obtained with the 7 images between May, 11th and July, 5th.

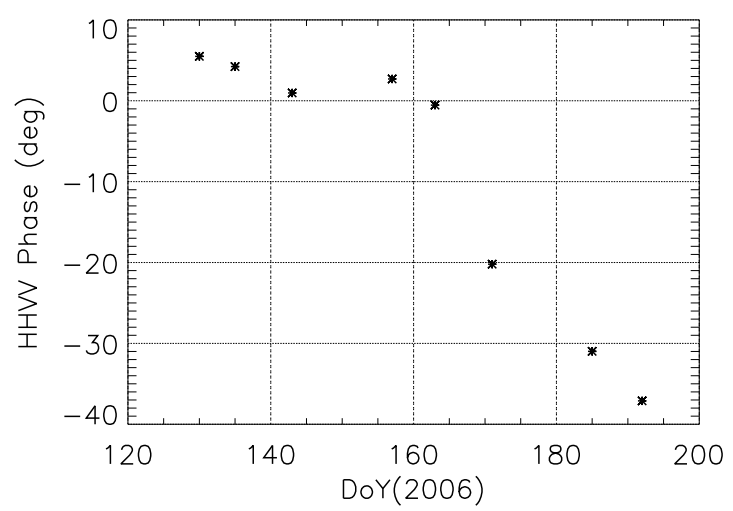

Fig. 9. The polarimetric phase between $\mathrm{HH}$ and VV channels. 
The moisture values are obtained minimizing the following figure of merit $F$ :

$$
F=\sum_{n>m>k}\left|\varphi_{n, m, k}-\varphi_{n, m, k}^{\prime}\right|_{2 \pi}^{2}+\sum_{n, k}\left|\gamma_{n, k}-\gamma_{n, k}^{\prime}\right|^{2} .
$$

The primed symbols are the observed quantities, whereas the unprimed ones are the corresponding quantities predicted by the forward model, which is a function of the moisture values. The phase differences are always expressed in the ambiguous interval $[-\pi, \pi]$ and the minimization is constrained to yield moisture values between 0 and 1 . The first moisture value of the series is forced at $10 \%$ since our current understanding is that this technique is unable to yield absolute moisture levels, but only relative changes. It is thus necessary to provide a starting value. This might be unnecessary in the presence of long series of observations, which could offer calibration opportunities.

Results for the inversion based on coherence magnitudes and phase triplets are reported in Fig. 10 for the $\mathrm{HH}$ and VV channels, although there is no significative difference between them. For comparison, in situ measurements are reported in Fig. 12.

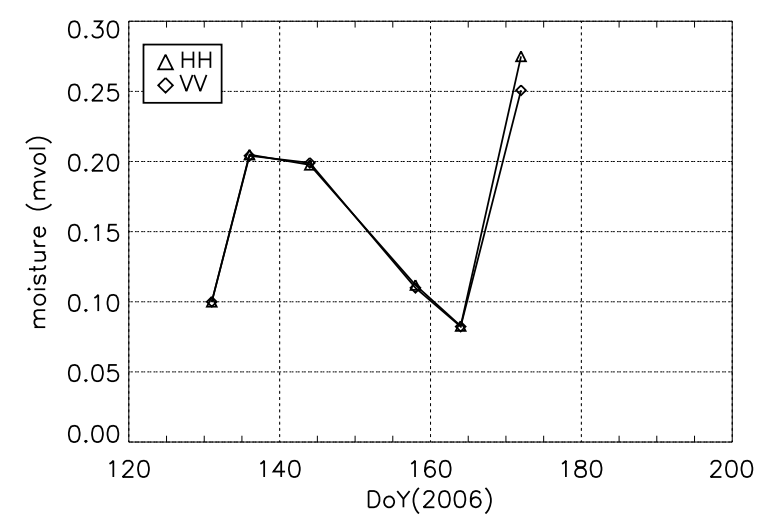

Fig. 10. Moisture from model inversion on field 222, supposed to be bare [16]. The first value is forced at $10 \%$. The averaging window is $50 \times 200$. The inversion algorithm is based on phase triplets and coherence magnitudes.

Other inversion possibilities involve using phase-only information, which might be more robust to temporal decorrelation. If the phases are well-calibrated, also with respect to motion, then they might be used directly, otherwise one could resort to the triplets, which are immune to any phase offset. Results based exclusively on the triplets are reported in Fig. 11.

In this preliminary inversion experiments, the results are comparable to in situ measurements. Apparently the tripletbased method is able to give good results also including the acquisition of July, 5th, which could be interpreted as an indication that phases are more robust than coherence magnitudes.

\section{Discussion}

The proposed model seems to fit well with experimental data in a particular case, however further investigations and

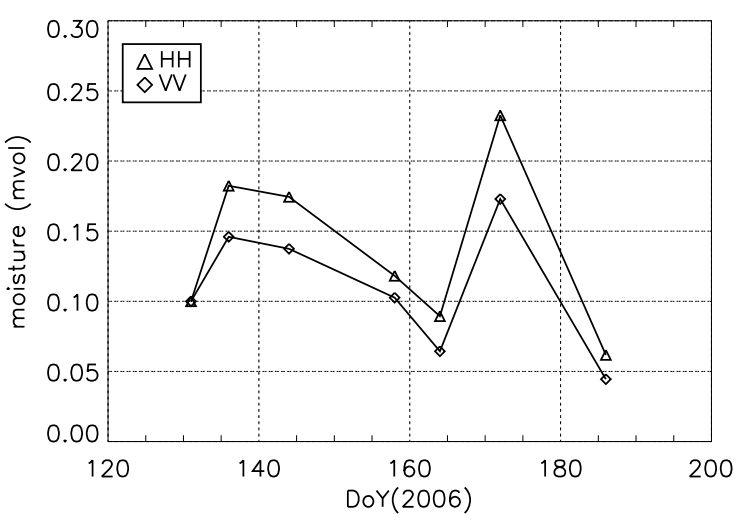

Fig. 11. Moisture from model inversion on field 222, supposed to be bare [16]. The first value is forced at $10 \%$. The averaging window is $50 \times 200$. The inversion algorithm is based on phase triplets.

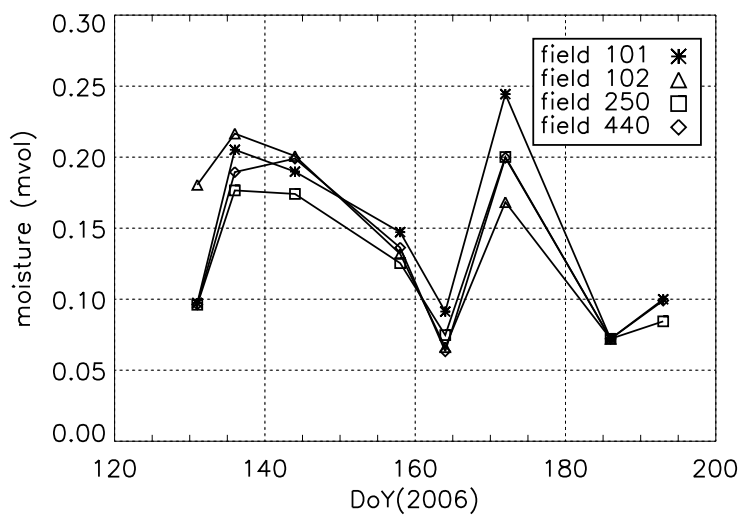

Fig. 12. In situ measurements of moisture in the top $5 \mathrm{~cm}$ of soil in fields nearby field 222. (Data provided by the European Space Agency, collected by the University of Kiel)

adaptations are needed to assess its validity beyond the given example.

One issue consists in the possible presence of surface roughness and the corresponding surface scattering component. Such component is expected to be less relevant for low frequencies and smooth surfaces, so that experiments with P-band would be very interesting. A surface component can affect both interferometric coherences and phases limiting the possibility of inversion. One might include it in the models, for instance resorting to polarimetric techniques and/or including amplitudes in the analysis. A similar discussion could be held for a vegetation component.

The penetration predicted by the proposed model is very limited: For example in L-band one finds two-way attenuations in the order of one to several decibels per centimeter (1$3 \mathrm{~dB} / \mathrm{cm}$ for moisture levels in the range 10\%-50\%). For this reason long wavelengths are interesting, since it is expected that they would be sensitive to moisture values in deeper layers.

Another issue involves the hypotheses of uniform scatterer density and uniform moisture in the vertical direction. The 
larger the penetration depth, the more questionable are the hypotheses. Different vertical scattering profiles are in principle easy to model with the Born approximation (integrating analytically or numerically Eq. 10). It is also relatively easy to model the phase variations in the vertical direction induced by a horizontal stratification of moisture. However it is unlikely that all these parameters can be inverted from the data, so that the choice of relevant parameters will be critical.

In this work we have been careful to select observables which are not sensitive to phase changes related to a classic deformation term. In this way we could avoid the need for accurate phase calibration; at the same time, we must say that our observations are not ruling out deformation as a possible additional explanation for phase changes. The observations of the interferogram mismatches exclude that the sole effect of moisture change is a rigid motion of the scattering layer.

\section{CONCLUSION}

A model based on plane waves and Born scattering is able to relate moisture variations to SAR interferometric observables, at least for bare fields acquired at L-band. According to this model, large phase and coherence effects can be simply explained with the differential vertical propagation of the electromagnetic waves, a function of different dielectric constants.

An unexpected property predicted by the model, the nonconservativeness of phases in triplets of images, has been observed in the data. This property might be used in the inverse problem, especially because it is insensitive to arbitrary phase offsets. On the other hand, its recognition calls for a revision of the optimal estimators derived for SAR interferometry with stacks (e.g. [17]), at least for some scenarios, taking into account non-real coherency matrices.

Vegetated soils require probably more complicated models: polarimetric techniques could help separating the various contributions.

Inversion of moisture variations from interferometric phases is likely to be feasible from a series of frequent observations, even though absolute figures might have to be obtained in a different way.

\section{ACKNOWLEDGMENT}

The authors would like to thank ESA (R. Bianchi) for providing validation data; the anonymous reviewers for their effort and constructive comments, which have made the paper less cryptical.

\section{REFERENCES}

[1] A. Gabriel, R. Goldstein, and H. Zebker, "Mapping small elevation changes over large areas: differential radar interferometry," J. Geophys. Res., vol. 94, no. B7, pp. 9183-9191, Jul. 1989.

[2] M. Nolan, D. R. Fatland, and L. Hinzman, "DInsar measurement of soil moisture," IEEE Trans. Geosci. Remote Sensing, vol. 41, no. 12, pp. 2802-2013, Dec. 2003.

[3] T. Zhang, Q. Zeng, Y. Li, and Y. Xiang, "Study on relation between InSAR coherence and soil moisture," in Proceedings of the ISPRS Congress, 2008, vol. 37, pp. $131-134$.

[4] B. Barrett, E. Dwyer, and P. Whelan, "The use of C- and L-band repeatpass interferometric SAR coherence for soil moisture change detection in vegetated areas," Open Remote Sensing Journal, vol. 5, no. 1, pp. 37 -53 , Jun. 2012
[5] I. Hajnsek and P. Prats, "Soil moisture estimation in time with DInSAR," Geoscience and Remote Sensing Symposium (IGARSS), IEEE International, vol. III, pp. 546-549, Jul. 2008.

[6] G. Nesti, D. Tarchi, and J.-P. Rudant, "Decorrelation of backscattered signal due to soil moisture changes," in International Geoscience and Remote Sensing Symposium, 1995, vol. 3, pp. 2026 - 2028.

[7] G. Nesti, D. Tarchi, D. Despan, J.-P. Rudant, A. Bedidi, P. Borderies, and E. Bachelier, "Phase shift and decorrelation of radar signal related to soil moisture changes," in Proc. of the 2nd International Workshop on Retrieval of Bio- \& Geo-Physical Parameter from SAR Data for Land Applications. 1998, pp. 423 - 430, ESA.

[8] S. Hensley, T. Michel, J. Van Zyl, R. Muellerschoen, B. Chapman, S. Oveisgharan, Z. S. Haddad, T. Jackson, and I. Mladenova, "Effect of soil moisture on polarimetric-interferometric repeat pass observations by UAVSAR during 2010 Canadian Soil Moisture campaign," Geoscience and Remote Sensing Symposium (IGARSS), IEEE International, pp. 1063 -1066 , Jul. 2011.

[9] K. Morrison, J.C. Bennett, M. Nolan, and R. Menon, "Laboratory measurement of the DInSAR response to spatiotemporal variations in soil moisture," Geoscience and Remote Sensing, IEEE Transactions on, vol. 49, no. 10 , pp. 3815 -3823, Oct. 2011.

[10] B. Barrett, E. Dwyer, and P. Whelan, "Soil moisture retrieval from active spaceborne microwave observations: An evaluation of current techniques," Remote Sensing, vol. 1, no. 3, pp. 210 - 242, Jul. 2009.

[11] B. Rabus, H. Wehn, and M. Nolan, "The importance of soil moisture and soil structure for InSAR phase and backscatter, as determined by FDTD modeling," IEEE Trans. Geosci. Remote Sensing, vol. 48, no. 5, pp. 2421-2429, May 2010.

[12] M. Woodward, Wave-equation tomography, Ph.D. thesis, Stanford, Dec. 1989.

[13] M.T. Hallikainen, F.T. Ulaby, M.C. Dobson, M.A. El-Rayes, and LilKun Wu, "Microwave dielectric behavior of wet soil - Part 1: Empirical models and experimental observations," Geoscience and Remote Sensing, IEEE Transactions on, vol. GE-23, no. 1, pp. 25 -34, Jan. 1985.

[14] S. Orfanidis, Electromagnetic waves and antennas, chapter 7, 2011, [available online at http://www.ece.rutgers.edu/ orfanidi/ewa/].

[15] C. Balanis, Antenna theory, John Wiley \& Sons, New York, 1997.

[16] DLR (German Aerospace Center), "AGRISAR 2006 Final Report," Tech. Rep., European Space Agency, Jan. 2008, [available online at http://earth.esa.int/].

[17] A. Monti Guarnieri and S. Tebaldini, "On the exploitation of target statistics for SAR interferometry applications," IEEE Trans. Geosci. Remote Sensing, vol. 46, no. 11, pp. 3436-3443, Nov. 2008.

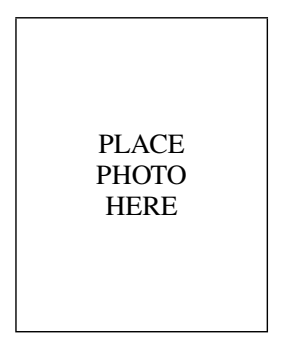

Francesco De Zan received his degree in Telecommunication Engineering from Politecnico di Milano (Italy) in 2004. From the same university he received the Ph.D. degree (2008), with a thesis on SAR interferometry and decorrelating targets. During his studies he has collaborated with T.R.E. (Milan, Italy) on persistent scatterer interferometry and with ESA about the Interferometric Wide Swath Mode of the Sentinel-1 mission. In 2007 he visited for six months the Stanford Exploration Project at Stanford University, California. He works since 2008 at the Microwaves and Radar Institute of DLR (German Aerospace Center), Oberpfaffenhofen, Germany. His interests include mission design and performance analysis of future interferometric SAR missions (e.g. BIOMASS, Tandem$\mathrm{L}$ ), interferometric/radargrammetric calibration of TanDEM-X, modeling and performance analysis for SAR interferometry and speckle tracking. 


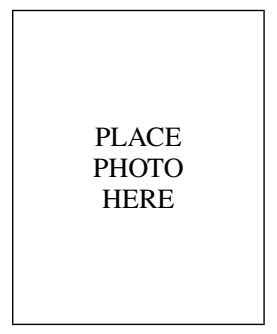

PSI Wide Area Processor.

Alessandro Parizzi received the master degree in Telecommunications Engineering from Politecnico di Milano in 2004. From 2004 to 2006 he was with the Electronic Department of Politecnico di Milano working in the field of SAR Interferometry. Since 2006 he has been with the Remote Sensing Technology Institute of the German Aerospace Center (DLR) in Oberpfaffenhofen, working on interferometric SAR processing. In the framework of the Terrafirma Project (ESA) he took part to the PSI Processing Validation and to the Development of the

\begin{tabular}{|c|}
\hline \\
PLACE \\
PHOTO \\
HERE \\
\end{tabular}

Pau Prats-Iraola (S'03-M'06) was born in Madrid, Spain, in 1977. He received the Ingeniero degree in telecommunication engineering and the Ph.D. degree from the Universitat Politècnica de Catalunya (UPC), Barcelona, Spain, in 2001 and 2006, respectively.

In 2001, he was a Research Assistant at the Institute of Geomatics, Spain. In 2002, he was at the Department of Signal Theory and Communications, UPC, where he worked in the field of airborne repeat-pass interferometry and airborne differential SAR interferometry. From December 2002 to August 2006, he was an Assistant Professor at the Department of Telecommunications and Systems Engineering, Universitat Autònoma de Barcelona, Barcelona,Spain. In 2006, he joined the Microwaves and Radar Institute, German Aerospace Center (DLR), Wessling, Germany, where, since 2009, he has been the Head of the Multimodal Algorithms Group. His research interests include airborne and spaceborne high-resolution SAR processing, SAR interferometry, differential SAR interferometry, and motion compensation for airborne systems.

Dr. Prats-Iraola was the recipient of the first prize of the Student Paper Competition of the 2005 IEEE International Geoscience and Remote Sensing Symposium (IGARSS), Seoul, Korea. In 2012, he was awarded with the DLR Science Award.

\begin{tabular}{|c|}
\hline \\
\\
PLACE \\
PHOTO \\
HERE \\
\end{tabular}

Paco López-Dekker (S'98-M'03) was born in Nijmegen, The Netherlands, in 1972. He received the Ingeniero degree in telecommunication engineering from the Universitat Politècnica de Catalunya (UPC), Barcelona, Spain, in 1997, the M.S. degree in electrical and computer engineering from the University of California, Irvine, in 1998, under the Balsells Fellowships, and the Ph.D. degree from the University of Massachusetts, Amherst, in 2003, for his research on clear-air imaging radar systems to study the atmospheric boundary layer. From 1999 to 2003, he was with the Microwave Remote Sensing Laboratory, University of Massachusetts. During 2003 he worked as a research scientist at Starlab, where he focused on the development of GNSS-R sensors. From 2004 to 2006, he was a Visiting Professor with the Telecommunications and Systems Engineering Department, Universitat Autonoma de Barcelona. In March 2006, he joined the Remote Sensing Laboratory, UPC, where he conducted research on bistatic synthetic aperture radar (SAR) under a five-year Ramon y Cajal Grant. At the university, he taught courses on signals and systems, signal processing, communications systems and radiation, and guided waves. Since November 2009, he leads the SAR Missions Group at the Microwaves and Radar Institute, German Aerospace Center, Oberpfaffenhofen, Germany. His current research focuses on the study of future SAR missions and novel mission concepts. 